\title{
Mesures de houles partiellement stationnaires en zones côtière et littorale
}

\author{
Vincent Rey ${ }^{1, a}$, Raphä̈l Certain ${ }^{2}$, Déborah Drevard ${ }^{1}$, Anne Meuret ${ }^{1}$ \\ ET JACQUES PIAZZOLA ${ }^{1}$ \\ 1 Université du Sud Toulon-Var, LSEET-LEPI, BP 56, 83162 La Valette du Var Cedex, France \\ 2 Université de Perpignan, LEGEM, 52 Av. P. Alduy, 66860 Perpignan, France
}

Reçu le 17 décembre 2004, accepté le 13 octobre 2005

\begin{abstract}
Résumé - Les instruments de mesures des courants en mer permettent d'obtenir un nombre d'informations de plus en plus important grâce à des techniques de mesures de plus en plus sophistiquées. En particulier, les mesures ponctuelles de vitesses et de pression permettent d'obtenir des informations sur l'énergie de la houle et sa direction de propagation. Nous avons montré dans ce travail que le caractère partiellement stationnaire des houles réelles peut être mesuré de façon quantitative à partir de données synchrones de vitesse et/ou de pression. Ces capacités sont illustrées par des exemples de mesures à partir de différents appareils immergés en zone littorale (S4, ADV, ligne de capteurs de pression).
\end{abstract}

Mots clés : Houle / réflexion / littoral / mesures

\begin{abstract}
Partially standing wave measurement in the nearshore. Instrumentation for field flow measurements is more and more sophisticated. In particular, local velocity and pressure measurements give informations on the wave energy and its propagation. In the present work, it is demonstrated that the use of coincident velocity and/or pressure data may give quantitative informations on the rate of standing waves. Examples from various instruments displayed in the nearshore are then given (S4, ADV, series of pressure sensors).
\end{abstract}

Key words: Water wave / reflection / nearshore / measurements

\section{Introduction}

Les données de houle à partir d'instruments en mer consistent généralement à recueillir des informations sur l'énergie transportée. Au large, l'énergie est répartie sur une gamme de fréquence plus ou moins large selon que la houle est formée ou confondue avec la mer de vent, et se propage autour d'une direction moyenne avec un angle d'ouverture qui dépend aussi de l'état de mer. De façon classique, la répartition énergétique obéit à des lois statistiques qui permettent de définir des paramètres caractéristiques qui sont la hauteur significative $H_{\mathrm{s}}$, la période significative $T_{\mathrm{S}}$ et la direction de propagation $\theta$.

À l'approche de la côte, la houle a tendance à « tourner par effets bathymétriques, et une dispersion apparaît dans la direction de propagation en fonction de la fréquence de chacune des composantes spectrales de la houle. De plus, pour des bathymétries abruptes, des

\footnotetext{
a Auteur correspondant : rey@univ-tln.fr
}

plages de forte pente, ou en présence de structures côtières (digues, épis...), une partie de l'énergie de la houle peut être réfléchie.

Si les caractéristiques de la houle au large sont maintenant bien connues et tout à fait utiles pour la navigation et le dimensionnement des ouvrages en mer, leur caractérisation au niveau du littoral reste plus difficile notamment d'une part pour des côtes accidentées aux bathymétries complexes et d'autre part pour les plages de sable où les facteurs morphologiques «transforment» de façon importante les conditions des houles incidentes du large jusqu'à leur déferlement.

Le but de ce travail est d'étudier les possibilités de mesure de houles multi-directionnelles et partiellement stationnaires à partir d'instruments donnant des informations sur les vitesses et/ou pression à profondeur donnée.

Après une description des modèles de houles puis les techniques d'analyses pour des houles uni- et multidirectionnelles, des applications in situ sont présentées et discutées. 


\section{Liste des paramètres}

\begin{tabular}{|c|c|}
\hline$C_{\varphi}$ & vitesse de phase de l'onde, en m.s ${ }^{-1}$ \\
\hline$C_{\mathrm{g}}$ & vitesse de groupe de l'onde, en m.s ${ }^{-1}$ \\
\hline$D$ & répartition angulaire de l'énergie de l'onde, sans unité $\left(\operatorname{rad}^{-1}\right)$ \\
\hline$E$ & énergie de l'onde, $E / \rho g$ en $\mathrm{m}^{2} \cdot \mathrm{Hz}^{-1}$ \\
\hline$E_{\mathrm{d}}$ & énergie par unité de fréquence et d'angle de l'onde, en $\mathrm{m}^{2} \cdot \mathrm{Hz}^{-1}$ \\
\hline$H$ & hauteur crête à creux de l'onde, en m \\
\hline$R$ & coefficient de réflexion de l'onde, sans unité \\
\hline$S$ & énergie par unité de fréquence de l'onde, en $\mathrm{m}^{2} \cdot \mathrm{Hz}^{-1}$ \\
\hline$T$ & période de l'onde, en s \\
\hline$a$ & amplitude de l'onde incidente, en $\mathrm{m}$ \\
\hline$b$ & amplitude de l'onde réfléchie, en m \\
\hline$f$ & fréquence de l'onde, en $\mathrm{Hz}$ \\
\hline$g$ & accélération due à la gravité, en $\mathrm{m} \cdot \mathrm{s}^{-2}$ \\
\hline$h$ & profondeur d'eau, en m \\
\hline$k$ & vecteur d'onde de l'onde, en $\mathrm{m}^{-1}$ \\
\hline$p$ & pression, en $\mathrm{Pa}$ \\
\hline$u$ & amplitude de la vitesse horizontale suivant l'axe $\mathrm{O} x$, en m.s ${ }^{-1}$ \\
\hline$u_{\mathrm{h}}$ & amplitude de la vitesse horizontale, en $\mathrm{m} . \mathrm{s}^{-1}$ \\
\hline$v$ & amplitude de la vitesse horizontale suivant l'axe $\mathrm{O} y$, en m.s ${ }^{-1}$ \\
\hline$w$ & amplitude de la vitesse verticale suivant l'axe $\mathrm{O} z$, en $\mathrm{m} . \mathrm{s}^{-1}$ \\
\hline$x, y$ & distances suivant les axes horizontaux $\mathrm{O} x$ et $\mathrm{O} y$, en $\mathrm{m}$ \\
\hline$z$ & distance suivant l'axe vertical $\mathrm{O} z$ orienté vers le haut, en $\mathrm{m}$ \\
\hline$\Delta$ & différence de phase entre les sondes, sans unité \\
\hline$\eta$ & déformée de la surface libre, en m \\
\hline$\eta_{\mathrm{i}}$ & déformée de la surface libre pour l'onde incidente, en m \\
\hline$\eta_{\mathrm{r}}$ & déformée de la surface libre pour l'onde réfléchie, en m \\
\hline$\varphi$ & phase de l'onde, sans unité \\
\hline$\phi$ & potentiel des vitesses, en $\mathrm{m}^{2} \cdot \mathrm{s}^{-1}$ \\
\hline$\lambda$ & longueur d'onde de l'onde, en m \\
\hline$\rho$ & masse volumique du fluide, en $\mathrm{kg} \cdot \mathrm{m}^{-3}$ \\
\hline$\theta$ & angle d'incidence de l'onde, sans unité (rad) \\
\hline$\omega$ & fréquence angulaire de l'onde, en rad.s ${ }^{-1}$ \\
\hline
\end{tabular}

\section{Théorie des ondes monochromatiques linéaires}

On se limite dans cette partie au cas bidimensionnel dans le plan $x \mathrm{O} z$, où $\mathrm{O} x$ représente l'axe horizontal, direction de propagation, et $\mathrm{O} z$, orienté vers le haut, l'axe vertical. Pour une houle ayant pour angle d'incidence $\theta$ par rapport à la direction $\mathrm{O} x$ dans le plan horizontal $x \mathrm{O} y$, il suffit de faire une rotation pour revenir au cas bidimensionnel. On peut noter que pour l'analyse des données de vitesses et de pression, l'hypothèse « onde plane » sera requise pour l'analyse des champs de vitesses et de pressions, ce qui signifie que les variations bathymétriques doivent être faibles dans la zone de mesure. On suppose que la houle est monochromatique, de fréquence $f$ et de période $T=1 / f$.

\subsection{Onde progressive}

Dans l'hypothèse d'ondes de faible amplitude de fréquence angulaire $\omega$, la dépendance vis-à-vis du temps du potentiel des vitesses et de la déformée de la surface libre peut s'écrire sous la forme complexe $\mathrm{e}^{\mathrm{i} \omega t}$. Les solutions pour une onde propagative (dans cette section, dans la direction $\mathrm{O} x$ pour simplifier) par profondeur constante $h$, satisfaisant l'équation de Laplace, les conditions limites au fond et les conditions linéarisées à la surface libre sont données par

$$
\phi(x, z, t)=\frac{a C(z)}{k} \mathrm{e}^{\mathrm{i}(\omega t-k x)}
$$

où $C(z)=\omega \frac{\cosh [k(z+h)]}{\sinh (k h)}$. Ceci correspond à une déformée de la surface libre

$$
\eta(x, t)=-i a \mathrm{e}^{\mathrm{i}(\omega t-k x)}
$$

où $H=2 a$ est la hauteur crête à creux. Le vecteur d'onde $k$ vérifie la relation de dispersion $\omega^{2}=g k \tanh (k h)$ où $g$ est l'accélération due à la gravité. L'onde se propage à la vitesse $C_{\varphi}=\omega / k$ et sa longueur d'onde $\lambda=$ $((2 \pi) / k)=C_{\varphi} T$ diminue quand la profondeur d'eau diminue. Les champs de vitesses sont donnés par :

$$
\begin{gathered}
u=\frac{\partial \Phi}{\partial x}=-i a C(z) \mathrm{e}^{\mathrm{i}(\omega t-k x)}=C(z) \eta \\
w=\frac{\partial \Phi}{\partial z}=a S(z) \mathrm{e}^{\mathrm{i}(\omega t-k x)}=i S(z) \eta
\end{gathered}
$$


avec $S(z)=\omega \frac{\sinh [k(z+h)]}{\sinh (k h)}$. Le champ de pression est donné par :

$$
p+\rho g z=-\rho \frac{\partial \Phi}{\partial t}=\frac{\rho \omega}{k} C(z) \eta
$$

L'énergie moyenne par unité de surface $\mathrm{d} x \mathrm{~d} y$ est $E=$ $\rho g a^{2} / 2$, le flux moyen d'énergie $E_{\mathrm{t}}$ à travers le plan $x \mathrm{O} y$ est donné par $E_{\mathrm{t}}=E C_{\mathrm{g}}$ où $C_{\mathrm{g}}=\partial \omega / \partial k$ est la vitesse de groupe. En supposant que l'on dispose des mesures $u^{(m)}=|u| \mathrm{e}^{\mathrm{i} \varphi_{u}}, w^{(m)}=|w| \mathrm{e}^{\mathrm{i} \varphi_{w}}$ à la profondeur $z$, et $p^{(m)}=|p| \mathrm{e}^{\mathrm{i} \varphi_{p}}$ à la profondeur $z_{\mathrm{p}}$, pour une abscisse $x$ fixée, l'amplitude $a$ peut donc être calculée avec l'une de ces trois données :

$$
a=\frac{|u|}{C(z)}=\frac{|w|}{S(z)}=\frac{k}{\rho \omega} \frac{|p|}{C\left(z_{\mathrm{p}}\right)}
$$

Si la pression mesurée est donnée en mètres par l'appareil, alors $p^{(m)}=|p| / \rho g$ et $|p|=\rho g\left|p^{(m)}\right|$. Un seul des trois paramètres suffit pour déterminer l'amplitude $a$.

\subsection{Onde partiellement stationnaire}

Pour une houle partiellement stationnaire, la déformée de la surface libre peut s'écrire

$$
\begin{aligned}
\eta(x, t) & =-i a \mathrm{e}^{\mathrm{i}(\omega t-k x)}-i b \mathrm{e}^{\mathrm{i}(\omega t+k x+\varphi)} \\
& =\eta_{\mathrm{i}}(x, t)+\eta_{\mathrm{r}}(x, t)
\end{aligned}
$$

où $\eta_{\mathrm{i}}$ et $\eta_{\mathrm{r}}$ correspondent respectivement aux ondes incidente et réfléchie. Dans ce cas, les composantes $u(x, z, t)$ et $w(x, z, t)$ de la vitesse et la pression $p(x, z, t)$, à une profondeur $z$ sont données par (en notation complexe)

$$
\begin{aligned}
& u=C(z)\left[\eta_{\mathrm{i}}-\eta_{\mathrm{r}}\right] \\
& w=i S(z)\left[\eta_{\mathrm{i}}+\eta_{\mathrm{r}}\right] \\
& p+\rho g z=\frac{\rho \omega}{k} C(z)\left[\eta_{\mathrm{i}}+\eta_{\mathrm{r}}\right]
\end{aligned}
$$

\subsubsection{Mesures synchrones de vitesses et de pression en un point donné d'abscisse $x$}

Des instruments permettant les mesures simultanées de vitesse horizontale et de pression [1] ou de la vitesse verticale (vélocimètre 3D, [2]) peuvent être utilisés pour la mesure de la réflexion. On remarque cependant que les équations (9) et (10) sont redondantes pour le calcul de $a$ et $b$. En utilisant $u^{(m)}=|u| \mathrm{e}^{\mathrm{i} \varphi_{u}}$ et $w^{(m)}=|w| \mathrm{e}^{\mathrm{i} \varphi_{w}}$, on peut écrire :

$$
\begin{aligned}
|u| \mathrm{e}^{\mathrm{i} \varphi_{u}} & =C(z)\left[-i a \mathrm{e}^{\mathrm{i}(\omega t-k x)}+i b \mathrm{e}^{\mathrm{i}(\omega t+k x+\varphi)}\right] \\
|w| \mathrm{e}^{\mathrm{i} \varphi_{w}} & =i S(z)\left[-i a \mathrm{e}^{\mathrm{i}(\omega t-k x)}-i b \mathrm{e}^{\mathrm{i}(\omega t+k x+\varphi)}\right]
\end{aligned}
$$

En prenant le module de l'expression obtenue en ajoutant l'équation (11) multipliée par $S(z)$ et l'équation (12) multipliée par $-i C(z)$, on obtient

$$
a=\frac{1}{2}\left[\frac{|u|^{2}}{C^{2}(z)}+\frac{|w|^{2}}{S^{2}(z)}+\frac{2|u||w|}{C(z) S(z)} \sin \left(\varphi_{w}-\varphi_{u}\right)\right]^{\frac{1}{2}}
$$

en multipliant cette fois l'équation (12) par $i C(z)$, on obtient

$$
b=\frac{1}{2}\left[\frac{|u|^{2}}{C^{2}(z)}+\frac{|w|^{2}}{S^{2}(z)}-\frac{2|u||w|}{C(z) S(z)} \sin \left(\varphi_{w}-\varphi_{u}\right)\right]^{\frac{1}{2}}
$$

En utilisant $u^{(m)}=|u| \mathrm{e}^{\mathrm{i} \varphi_{u}} \quad$ (profondeur $z$ ) et $p^{(m)}=$ $|p| \mathrm{e}^{\mathrm{i} \varphi_{p}}$ (profondeur $z_{\mathrm{p}}$ ), on obtient par un calcul similaire

$$
\begin{aligned}
a=\frac{1}{2}\left[\frac{|u|^{2}}{C^{2}(z)}+\right. & \frac{k^{2}}{\rho^{2} \omega^{2}} \frac{|p|^{2}}{C^{2}\left(z_{\mathrm{p}}\right)} \\
& \left.+\frac{2|u||p|}{C(z) C\left(z_{\mathrm{p}}\right)} \frac{k}{\rho \omega} \sin \left(\varphi_{\mathrm{p}}-\varphi_{u}\right)\right]^{\frac{1}{2}}
\end{aligned}
$$

$$
\begin{aligned}
b=\frac{1}{2}\left[\frac{|u|^{2}}{C^{2}(z)}+\right. & \frac{k^{2}}{\rho^{2} \omega^{2}} \frac{|p|^{2}}{C^{2}\left(z_{\mathrm{p}}\right)} \\
& \left.-\frac{2|u||p|}{C(z) C\left(z_{\mathrm{p}}\right)} \frac{k}{\rho \omega} \sin \left(\varphi_{\mathrm{p}}-\varphi_{u}\right)\right]^{\frac{1}{2}}
\end{aligned}
$$

On peut noter que pour des ondes d'incidence oblique, une hypothèse doit être faite sur la direction de l'onde incidence et/ou sur l'angle entre ondes incidente et réfléchie (voir par exemple [1-3]).

\subsubsection{Mesures synchrones de pressions en des points donnés d'abscisses différentes}

Après transformée de Fourier des données de pression mesurées aux positions $x_{n}$, et à la profondeur $z$, l'amplitude «mesurée » pour la fréquence $f=2 \pi \omega$ peut être écrite en fonction de la pression mesurée $p^{(m)}$ à cette même abscisse selon

$$
\eta_{n}^{(m)}=\frac{k}{\rho \omega C\left(z_{p}\right)} p^{(m)}=A_{n} \mathrm{e}^{\mathrm{i} \varphi_{n}}
$$

Si on considère 2 sondes respectivement en $x_{1}$ et $x_{2}$, la différence de phase entre les sondes est donnée par $\Delta=$ $k\left(x_{2}-x_{1}\right)$ avec $k$ le vecteur d'onde dans cette direction. Si on écrit $\varphi_{2}=\varphi_{1}+\delta$, et $\eta=\eta_{1}^{(m)}$ pour $x=x_{1}, \eta=\eta_{2}^{(m)}$ pour $x=x_{2}$, on obtient les coefficients des ondes incidente et réfléchie

$$
\begin{aligned}
& a=\frac{1}{2|\sin \Delta|}\left[A_{1}^{2}+A_{2}^{2}-2 A_{1} A_{2} \cos (\delta+\Delta)\right]^{\frac{1}{2}} \\
& b=\frac{1}{2|\sin \Delta|}\left[A_{1}^{2}+A_{2}^{2}-2 A_{1} A_{2} \cos (\delta-\Delta)\right]^{\frac{1}{2}}
\end{aligned}
$$


Si on considère 3 sondes respectivement en $x_{1}, x_{2}$ et $x_{3}$, on définit $\Delta_{n}=k\left(x_{n}-x_{1}\right)$, et $\varphi_{n}=\varphi_{1}+\delta_{n}, n=1,2,3$, les modules des amplitudes $a$ et $b$ sont données par Goda et al. (1976) [4], Rey et al. (2002) [5] :

$$
|a|=\left|\frac{s_{2} s_{3}-3 s_{4}}{s_{5}}\right|, \quad|b|=\left|\frac{s_{1} s_{4}-3 s_{3}}{s_{5}}\right|
$$

où

$$
\begin{aligned}
& s_{1}=\sum_{n=1}^{3} \mathrm{e}^{-2 \mathrm{i} \Delta_{n}}, \quad s_{2}=\sum_{n=1}^{3} \mathrm{e}^{2 \mathrm{i} \Delta_{n}}, \quad s_{3}=\sum_{n=1}^{3} \mathrm{e}^{-\mathrm{i}\left(\Delta_{n}+\varphi_{n}\right)}, \\
& s_{4}=\sum_{n=1}^{3} \mathrm{e}^{\mathrm{i}\left(\Delta_{n}+\varphi_{n}\right)}, \quad s_{5}=s_{1} s_{2}-9
\end{aligned}
$$

Le coefficient de réflexion est alors défini par $R=|b / a|$.

\subsection{Houles réelles}

Les états de mer réels sont souvent considérés comme des états aléatoires et les quantités utilisées pour décrire ces états de mer sont par exemple la hauteur moyenne des vagues, les hauteur et période significatives, etc. Ces paramètres sont calculés à partir d'analyses statistiques de périodogrammes ou de spectres respectivement pour les domaines temporel et fréquentiel. Nous utilisons ici la méthode couramment utilisée basée sur l'analyse de Fourier du signal (analyse spectrale), elle suppose une représentation des houles réelles par une superposition de houles d'Airy (approximation linéaire). Les vitesses pour chacune des composantes spectrales sont $u^{(m)}=|u| \mathrm{e}^{\mathrm{i} \varphi_{u}}$, $v^{(m)}=|v| \mathrm{e}^{\mathrm{i} \varphi_{v}}, w^{(m)}=|w| \mathrm{e}^{\mathrm{i} \varphi_{w}}$. Elles sont calculées par transformée de Fourier rapide (FFT). Pour chaque composante spectrale de fréquence $f$, on calcule la direction de l'onde et l'intensité de la vitesse horizontale $u_{\mathrm{h}}$

$$
\begin{aligned}
& \theta(f)=\arctan \left(\frac{|v|}{|u|}\right) \text { si }|u| \neq 0 \text { et } \theta=\frac{\pi}{2} \text { si }|u|=0 \\
& \left|u_{\mathrm{h}}\right|^{2}=|u|^{2}+|v|^{2}
\end{aligned}
$$

Le spectre directionnel $E_{\mathrm{d}}(f, \theta)=S(f) D(\theta, f)$ dépend de la fréquence en particulier par profondeur finie. Nous nous limitons à la direction de propagation moyenne $\theta(f)$ pour estimer $D(\theta, f)$. En supposant que la pente du fond est faible, l'approximation onde plane peut être appliquée. Le spectre énergétique basé sur une onde propagative ou partiellement réfléchie peut être calculé en utilisant les formules présentées dans les sections 2.1 et 2.2. Les spectres énergétiques montrés dans la section suivante ont été lissés par une moyenne sur les fréquences.

\section{Applications}

L'instrumentation classique donne des informations soit sur les déplacements de la surface libre (déformation de la surface libre et accélérations horizontales pour les bouées en pilonnement-roulis), soit sur le mouvement dans le fluide (vitesses et pression). Les instruments peuvent être utilisés de façon indépendante ou synchrone, selon la quantité d'informations requises et les contraintes expérimentales. Les bouées multi-directionnelles sont équipées d'accéléromètres tridimensionnels. Après double intégration des signaux, le spectre énergétique peut être déduit de la composante verticale, tandis que les composantes horizontales permettent de déterminer la direction principale de la houle (à $\pi$ près) et sa répartition angulaire. En eau peu profonde, on utilise plutôt des capteurs de pression. Des instruments plus récents, comme l'ADV (vélocimètre acoustique doppler) ou l'ADCP (profileur acoustique doppler), ou le S4 (de principe basé sur la mesure des vitesses à partir de la déformation d'un champ magnétique) sont utilisés en zones côtière et littorale pour les mesures de vitesses (et de pression). Pour les houles multidirectionnelles, souvent observées en zone littorale, on peut donc s'attendre à des données plus précises concernant les répartitions angulaires, à partir des instruments de mesures des vitesses horizontales. En eau peu profonde ou pour les expériences en laboratoire, les calculs de directions peuvent être facilités par l'utilisation de réseaux de capteurs (sondes à houle, capteurs de pression). Les deux exemples présentés ici ont pour but d'illustrer la capacité d'ADV et $\mathrm{S} 4$, et des capteurs de pression à mesurer des houles partiellement stationnaires in situ.

\subsection{Campagnes expérimentales de Carqueiranne}

Ces campagnes de mesures ont été effectuées avec l'entreprise Thetis en juin 2001 et mai 2002 [6]. Elles ont montré un bon accord pour les données significatives de houle basées sur des ondes progressives [6]. L'ADV, disposé sur un support immergé par 9,65 m de fond, mesurait les vitesses horizontales à une profondeur $z=8,70 \mathrm{~m}$ et la pression à la profondeur $z_{\mathrm{p}}=9,20 \mathrm{~m}$ de fond. La fréquence d'acquisition était de $8 \mathrm{~Hz}$. On a représenté sur la figure 1 le spectre énergétique obtenu à partir des mesures soit de la vitesse horizontale, soit de la vitesse verticale, soit de la pression. On observe que les résultats sont très similaires pour la vitesse horizontale et la pression. Le résultat calculé à partir de la vitesse verticale est très bruité.

Ceci est dû au fait que l'appareil est quasiment au fond, ce qui correspond à une vitesse verticale très faible d'après la condition d'imperméabitité, vitesse en outre très bruitée à cause des perturbations apportées par la présence de l'appareil et de son support. On a ensuite séparé à partir des données combinées de la vitesse horizontale et de la pression les composantes incidente et réfléchie de la houle. On observe sur la figure 2 que la houle est quasiment progressive (réflexion inférieure à $5 \%$ ), et on retrouve par conséquent la répartition énergétique présentée sur la figure 1 pour les calculs basés respectivement sur les données de vitesses horizontale et de pression. La figure 3 montre la direction de propagation 


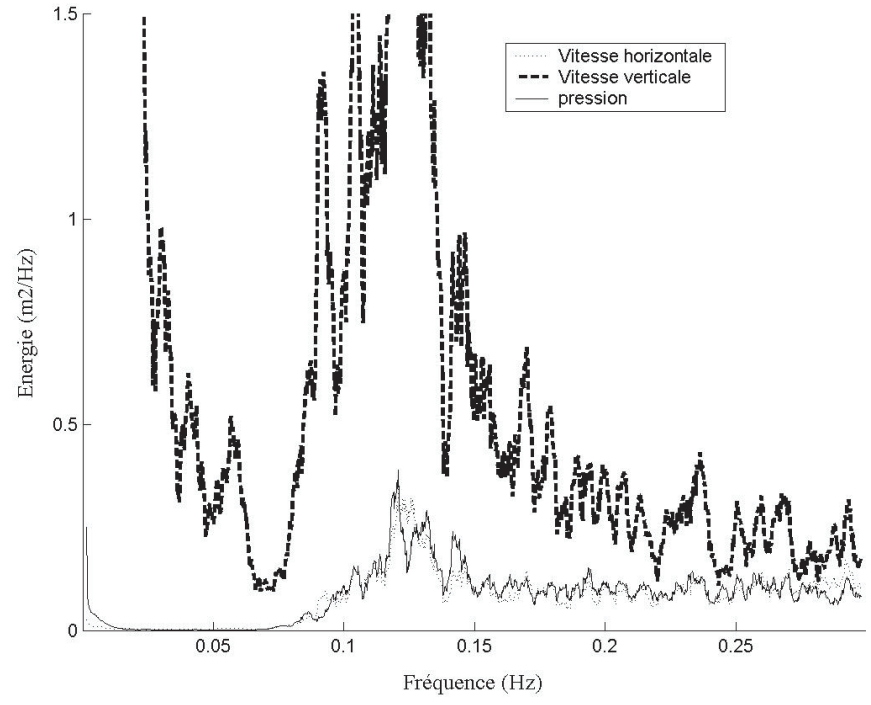

Fig. 1. Spectre d'énergie, calcul dans l'hypothèse d'une houle progressive.

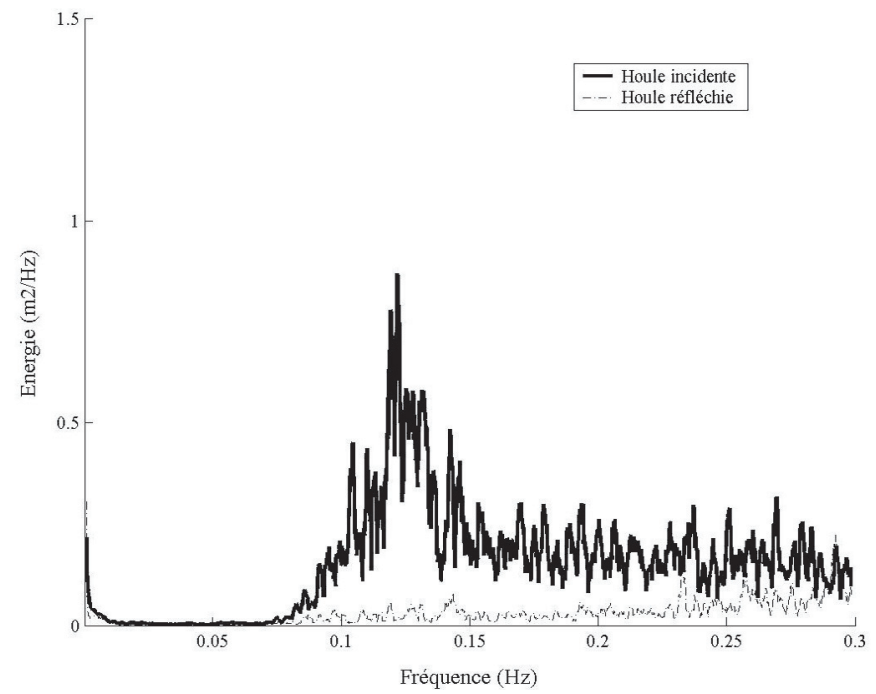

Fig. 2. Spectre d'énergie, calcul dans l'hypothèse d'une houle partiellement stationnaire.

en fonction de la fréquence, elle correspond à une houle de direction ouest sud-ouest. Notons que l'algorithme de calcul basé sur une houle partiellement stationnaire permet de lever l'indétermination d'un angle $\pi$ sur le sens de propagation. Cette information peut s'avérer utile pour des zones où ni les données climatiques de vent, ni la configuration du site ne permettent d'écarter un des deux sens de propagation.

\subsection{Campagne expérimentale de Sète}

Des expériences ont été menées sur la plage à barres de Sète durant un mois en novembre 2000 dans le but d'étudier les effets de la houle sur la formation et la migration des barres sédimentaires sous-marines [7]. Trois instruments $\mathrm{S} 4$ étaient disposés respectivement au niveau

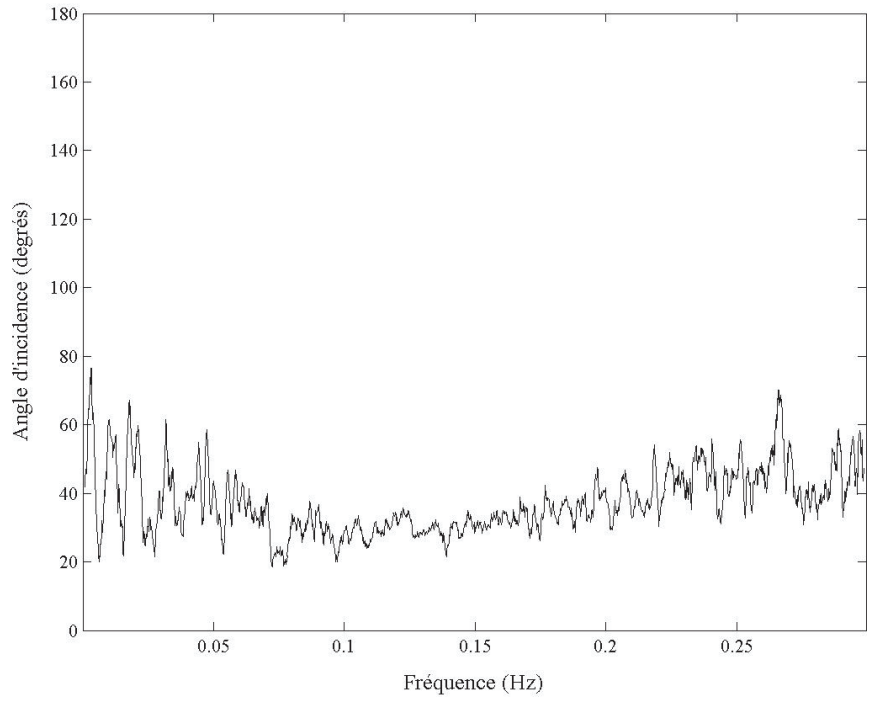

Fig. 3. Direction de propagation de la houle incidente en fonction de la fréquence.

de la fosse interne, de la fosse externe, et au large de la deuxième barre. Par ailleurs, une série de cinq capteurs de pression, distants d'environ $10 \mathrm{~m}$ les uns des autres, était alignée dans la direction perpendiculaire à la côte, au niveau du sommet de la barre interne. Les détails d'expériences sont présentés dans [8]. Nous nous limitons ici à des résultats significatifs des possibilités des instruments à mesurer des houles partiellement réfléchies.

Lors d'épisodes de tempêtes, on a pu observer [3] que la plage est dissipative et que la houle est progressive. À titre d'exemple, on a représenté sur les figures 4 et 5 les spectres d'énergies à partir des données de vitesse horizontale et de pression du S4 situé le plus au large par $6 \mathrm{~m}$ de fond. On observe sur la figure 4 des données de répartition d'énergie à partir des calculs basés sur des ondes progressives similaires, que ce soit à partir des données de vitesse ou de pression. Le calcul basé sur une onde partiellement stationnaire et présenté sur la figure 5 confirme la répartition spectrale de la figure 4, toute l'énergie étant transportée par l'onde incidente.

Nous avons ensuite étudié les spectres pour un épisode de calme relatif, caractérisé par la présence d'ondes assez longues de faible amplitude. Les données des trois S4 s'accordent à montrer une composante réfléchie notable, et à peu près identique pour les trois $\mathrm{S} 4$, et qui est attribuée à une réflexion par le haut de plage [3]. À titre d'exemple, on a représenté sur les figures 6 et 7 les résultats basés sur des ondes partiellement stationnaires, respectivement pour le S4 le plus au large et pour les trois capteurs de pression les plus près de la plage, distants respectivement de 8,5 et $10,5 \mathrm{~m}$.

On observe une réflexion de l'ordre de $30 \%$, ce qui est assez conséquent pour le $\mathrm{S} 4$, celle mesurée à partir des capteurs de pression est cependant plus faible, de l'ordre de $15 \%$, mais non négligeable. Ceci peut s'expliquer par des mesures en des zones différentes du site d'étude, dont la bathymétrie présente un aspect tridimensionnel. 


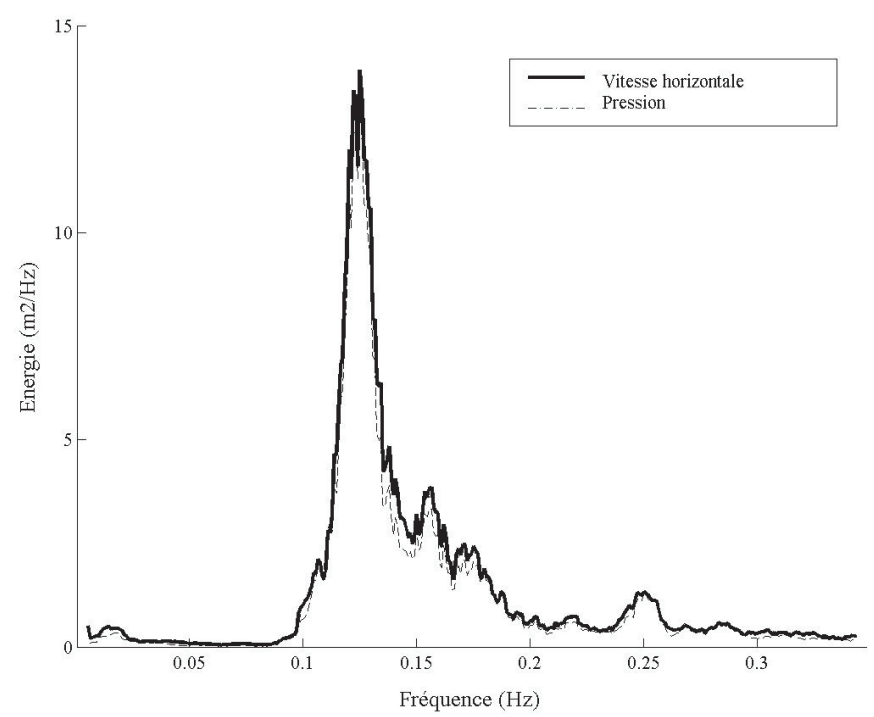

Fig. 4. Spectre d'énergie, épisode de tempête, houle progressive.

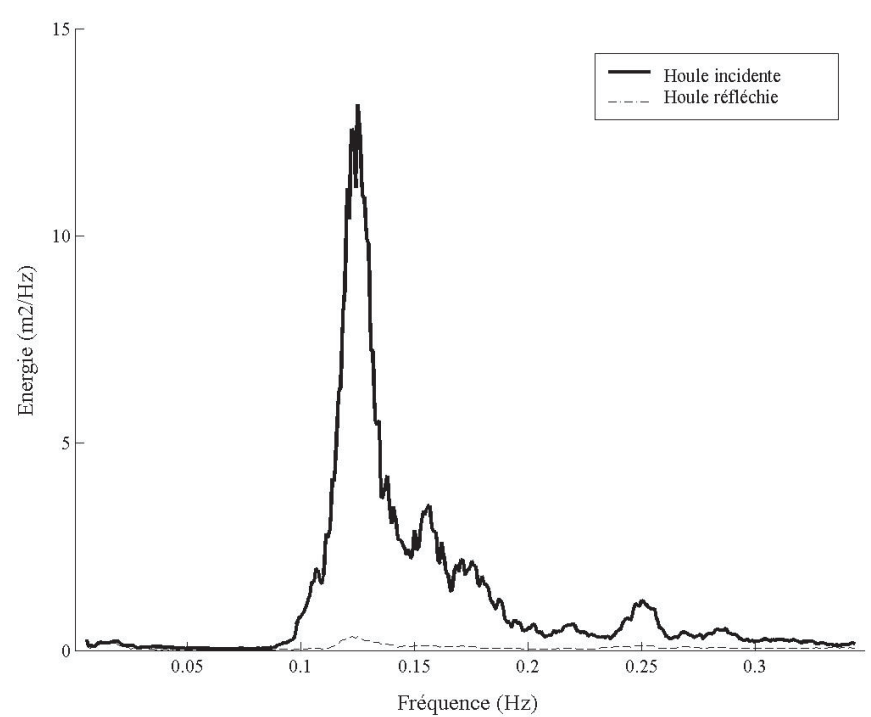

Fig. 5. Spectre d'énergie, épisode de tempête, houle partiellement stationnaire.

\section{Conclusions}

Les études des houles en zone littorale présentent un intérêt important pour la compréhension de la dynamique littorale, avec en particulier les problèmes d'érosion des plages. De façon plus appliquée, la connaissance de l'hydrodynamique est indispensable pour un dimensionnement adéquat des structures côtières, et les études d'impact d'ouvrage sur l'équilibre dynamique du littoral. Les données de houles sont primordiales, tant pour leur action directe sur les ouvrages ou le fond que pour les courants et le transport sédimentaire associé qu'elles induisent.

À l'heure actuelle, et en complément des modèles numériques développés, les mesures sont toujours autant nécessaires et les nouvelles techniques instrumentales permettent d'obtenir un nombre d'informations de

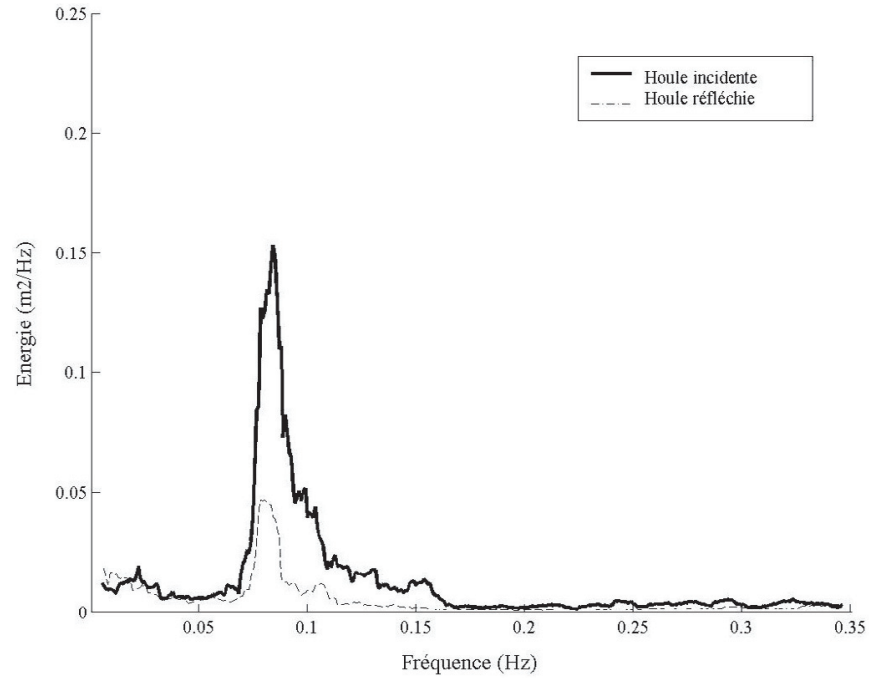

Fig. 6. Spectre d'énergie (S4), épisode de calme relatif, houle partiellement stationnaire.

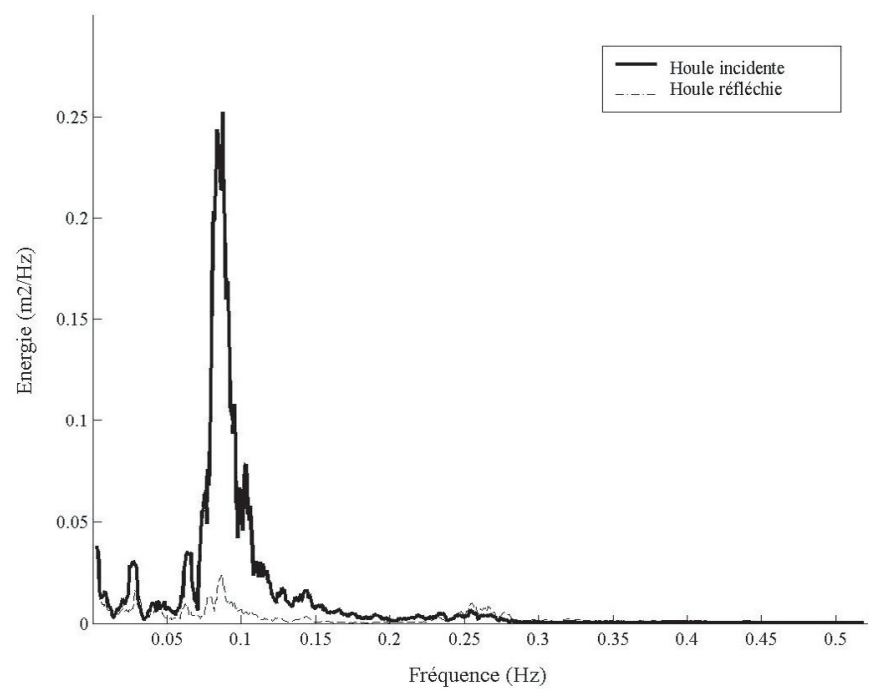

Fig. 7. Spectre d'énergie (capteurs de pression), épisode de calme relatif, houle partiellement stationnaire.

plus en plus important. Nous avons montré dans ce travail que le caractère partiellement stationnaire des houles réelles peut être quantifié, ce qui présente un intérêt particulier pour l'étude de plages réflectives ou d'agitation au voisinage de structures. L'utilisation d'appareils de mesures profileurs de vitesses pour des données plus complètes de courants, de houles ainsi que de turbulence sont envisagées à court terme en complément des appareils présentés.

Remerciements. Les auteurs remercient la Société Thétis SA pour les expériences menées à Carqueiranne dans le cadre du travail de thèse de Anne Meuret (co-financement Région PACA - Société Thétis). La campagne expérimentale de Sète a été menée dans le cadre du Programme National PNEC ART 7. Les auteurs remercient son support financier et les participants à la campagne. 


\section{Références}

[1] T.L. Walton Jr, Wave reflection from natural beaches, Ocean Engineering 19 (1992) 239-258

[2] D. Drevard, A. Meuret, V. Rey, J. Piazzola, A. Dolle, Partially reflected waves measurements using Acoustic Doppler Velocimeter (ADV), Thirteenth International Offshore and Polar Engineering Conference ISOPE'03, Honolulu, Hawaii, 25-31 May 2003

[3] R. Certain, S. Meulé, V. Rey, C. Pinazo, Swell transformation on a microtidal barred beach (Sète, France), 2004, soumis à J. Marine Systems

[4] Y. Goda, Y. Suzuki, Estimation of incident and reflected waves in random wave experiments, Proceedings of the 15th Coastal Engineering Conference 1 (1976) 828-845
[5] V. Rey, R. Capobianco, C. Dulou, Wave scattering by a submerged plate in presence of a steady uniform current, Coastal Engineering 47 (2002) 27-34

[6] A. Meuret, Étude d'un système PUVW pour les mesures de houle et de turbulence en milieu littoral et côtier, Thèse de Doctorat, Univ. du Sud Toulon-Var, 2004, p. 170

[7] R. Certain, J.P. Barusseau, R. Capobianco, A. Meuret, V. Rey, C. Dulou, A. Stepanian, F. Levoy, H. Howa, Bottom and shoreline evolutions under wave actions at a french Mediterraneen site: The beach of Sète, MEDCOAST 2001, Hamammet, Tunisie, October 2001.

[8] R. Certain, Morphodynamique d'une côte sableuse microtidale à barres : le golfe du Lion (Languedoc-Roussillon), Thèse de Doctorat, Univ. Perpignan, 209 p., 2002 